\title{
Persistent longstanding illness and non-drinking over time, implications for the use of lifetime abstainers as a control group
}

Linda Ng Fat ${ }^{1}$, Noriko Cable ${ }^{1}$, Nicola Shelton ${ }^{1}$, MG Marmot ${ }^{1}$

'Department of Epidemiology and Public Health, UCL, 1-19 Torrington Place,

London, WC1E 6BT, England, PHONE NUMBER

Linda Ng Fat, Doctoral Student

Noriko Cable, Senior Research Fellow

Nicola Shelton, Senior Lecturer

Michael Marmot, Director: Institute of Health Equity

Key terms: ALCOHOL, LONGITUDINAL STUDIES, J-SHAPE, HEALTH

BEHAVIOUR, NON-DRINKERS

Word count:3,196

Correspondence to: Linda Ng Fat I.ngfat.10@ucl.ac.uk 


\section{Persistent longstanding illness and non-drinking over time, implications for the use of lifetime abstainers as a control group}

\section{Background}

Non-drinkers are shown to have worse health than moderate drinkers in later life. We examine the preceding health status of non-drinkers in early adulthood and whether persistent poor health is associated with persistent non-drinking

\section{Methods}

Using two prospective British birth cohort studies established in 1958 (NCDS) and in 1970 (BCS) participants who reported 'never' or 'never had an alcoholic drink' to drinking status questions in successive waves from 23 and 26 years in NCDS/BCS were derived as 'Lifetime abstainers'

Logistic regression on the odds of being a lifetime abstainer was carried out on changes in limiting longstanding illness (LLSI) in the NCDS and longstanding illness (LSI) in the BCS, adjusting for sex, education, poor psychosocial health, marital and parental status.

\section{Results}

Participants with a LLSI in consecutive waves since 23 years had 4.50 times the odds of someone who did not have a LLSI of being a lifetime abstainer at 33 years $(95 \% \mathrm{Cl} 1.99$ to 10.18) and 7.02 times the odds at 42 years (2.39 to 20.66) after adjusting for all factors. Similarly in the BCS having a LSI in consecutive waves resulted in higher odds of being a lifetime abstainer at 30 years (OR 2.80, 1.88 to 4.18 ) and 34 years (OR $3.33,2.01$ to 5.53 ) 


\section{Conclusion}

Persistent LSI was associated with remaining a non-drinker across adulthood. Studies comparing the health outcomes of moderate drinkers to lifetime abstainers that do not account for pre-existing poor health may overestimate the better health outcomes from moderate alcohol consumption.

Word count: 2,856

\section{Introduction}

Evidence for the $\mathrm{J} / \mathrm{U}$-shape relationship between alcohol consumption and mortality and morbidity, where non-drinkers and heavy drinkers have worse health than moderate drinkers in later life is established in the literature. Whilst this relationship appears among diverse populations and through time (1) and in various conditions such as coronary heart disease (2), cardiovascular disease (3), all-cause mortality (4) and more recently cognitive decline (5)whether the better health outcomes of moderate drinkers compared to non-drinkers can be completely attributable to moderate alcohol consumption has been debated.

The most prominent criticism was first proposed by Shaper and Colleagues in 1988 (6) which argues that many non-drinkers in later life have stopped drinking due to illness thus it is their pre-existing poor health which exaggerates the poor health outcomes of non-drinkers relative to drinkers, sometimes referred also to as 'sick-quitters'. Indeed ex-drinkers have higher rates of doctor-diagnosed illnesses including heart disease (7) and having a diagnosis of a chronic conditions was associated with increased probability of reduction or cessation of alcohol consumption $(8,9)$.

However poor health was also found to be associated with non-drinking among young adults (10), even after adjusting for a range of social and demographic factors (11). This raises the question as to whether poor health may precede non-drinking at the very onset of one's drinking trajectory and whether continued poor health is a reason for refraining from drinking across the life-course. If this is the case then the worse health of non-drinkers in later life 
may suffer from double bias, firstly from 'sick-quitters' and secondly from those who abstain throughout life due to continual poor health indicating further bias among lifelong abstainers. We call the later 'sick non-starters' which is the subject of investigation in this study through using two nationally representative prospective British cohort studies to assess whether persistent poor health is associated with remaining a non-drinker from early adulthood

\section{Method}

\section{Survey design}

The National Child Development Study 1958 (NCDS) and the British Cohort Study 1970 (BCS) are prospective cohorts studies established on around 17000 British babies born in their corresponding years. Data in the NCDS has been collected when participants were aged $7,11,16,33,42,46$ and 50 . Similarly data from the BCS was collected at birth and at age $5,10,16,26,30,34$ and 38 . In each cohort data has been collected from a variety of sources including parents, medical examiners and schools covering a range of areas including socioeconomic status and health. We use NCDS data collected at age 16 (1974) 23 (1981), 33 (1991) and 42 (2000), and in the BCS data collected at age 26 (1996), 30 (2000) and 34 (2004) since drinking frequency questions were not asked at 38 years. Our reason for using two cohorts is to validate the hypothesis that persistent self-reported illness is associated with persistent non-drinking since this hypothesis has not been explored before rather then compare effects between two cohorts. The different time points and measurements used in each cohort would also make it difficult to make a direct comparison.

\section{Measurements}

\section{Alcohol consumption}

In each of the specified waves participants were asked "How often do you usually have an alcoholic drink of any kind?" We derived a lifetime abstainer variable using a bottom up approach by netting together all those who said "Never nowadays" or "Never had an 
alcoholic drink" at 23 and 33 years, and in the second model up to 42 years. The same method was applied in the BCS using "Never nowadays" or "Never had an alcoholic drink" answers from 26 to 30 years, and in the second model up to 34 years. These two options "Never nowadays" and "never had an alcoholic drink" are provided to distinguish between non-drinkers and lifetime abstainers. To avoid confusion we refer to people who stated "never nowadays" as non-drinkers and the later as self-reported lifetime abstainers.

We also compare findings with self-reported lifetime abstainers those who stated "Never had an alcoholic drink" using the BCS at 30 and 34 years. This is not done in the NCDS as this option is not provided at 33 years. This method of deriving a lifetime abstainer from participants self-report is what is commonly used in the literature however two studies have found that half of participants who claimed to have never drank alcohol reported drinking in the previous waves of the surveys $(12,13)$. Whilst the self-report measure in the BCS appeared to be more accurate, with around $22 \%$ of self-reported lifetime abstainers at 34 years drinking in the past survey, there may be a validity issue with this self-report measure, thus we also use a bottom up approach by taking consistent 'never nowadays' answers as a different measure of being a persistent non-drinker. The percentage of lifetime abstainers derived using the bottom up approach ranged from 1 to $2.1 \%$ in NCDS and BCS. This did not differ greatly from self-reported lifetime abstainers alcohol in the BCS at $30(2.1 \%)$ and 34 years $(2.2 \%)$.

\section{Early health}

In the NCDS a local authority medical officer collected extensive data on the medical history of the participants when they were 16 years. Here a systematic examination was carried out on details of skin conditions, respiratory tract infections, and defects in the cardiovascular system, the alimentary tract, the urogenital system, hernias, bones and joints and the neuromuscular system. We converted each condition into a binary variable representing whether the condition was present (no disability to degree unknown) or not. We also created 
another binary variable to model whether a participant had at least one slight to severe disability or not.

\section{Longstanding and limiting longstanding illness}

Binary variables derived from yes/no answers to the question "Do you have any longstanding illness, disability or infirmity which limits your activities in any way compared with people of your own age?" are used to measure health status across different time waves by creating a four categorical variable as has done with self-rated poor health in a different longitudinal study (14). At age 33 this included all those who did not have a LLSI at age 23 and 33 as the reference category. The other three categories included those who had a LLSI at age 23 but not at 33 , those who had a LLSI at age 33 but not at age 23 , and finally all those who had a LLSI in in both waves. To combine LLSI information from age 23, 33 and 42 we reduced the three binary variables to four categories to keep degrees of freedom within the model. This includes two categories, one with participants who did not have a LLSI at age 42 but had a LLSI at age 23 or 33 , and the other with participants who had a LLSI at age 42 who may or may not have had a LLSI at age 23 or 33 . The reference and the final category are those who did not have a LLSI and had a LLSI in successive waves respectively.

The same variable derivation was applied to the BCS at age 26, 30 and 34, however with longstanding illness (LSI) as it was asked consistently in each wave (in the NCDS only LLSI was asked consistently), LSI was derived through yes responses to the question "Do you have any long-standing illness, disability or infirmity? By long-standing I mean anything that has troubled you over a period of time?" There is substantial overlap between LLSI and LSI, with LLSI illness questions often being asked only to respondents who said yes to have a LSI. In the NCDS for example, $65.4 \%$ of respondents with a LSI said that this was LLSI at 33 years when both questions was asked. 


\section{Controls}

All models are adjusted for self-report measures of sex, highest qualification, marital status, children under 16 years in the household, and poor mental health indicated by the malaise inventory score using the full 24 item scale categorised into normal (0-7), high (8-24) which has been found to be a valid measurement of psychosocial health (15). At age 34 in the BCS, only 9 of the original 24 items were asked resulting in a $4+$ cut off for depression. We used the most recent responses at the corresponding age of the model in both cohorts. For example for lifetime abstainers' age 33 in NCDS we used the highest qualification as recorded at age 33.

\section{Statistical Analysis}

Chi-squared tests was carried out on items from the medical examination at age 16 by drinking status at age 23 in the NCDS to observe whether each medical problem at an earlier age was associated with drinking in young adulthood.

Logistic regression on the odds of being a lifetime abstainer is used to explore whether remaining in ill health is associated with remaining a non-drinker, by examining associations with LLSI or LSI status between waves in NCDS and BCS, adjusting for sex, malaise inventory score, highest qualification, marital status and children in the household,, recorded in the most recent survey at the corresponding age of the model.

\section{Results}

Figure 1 shows achieved sample at each wave. In the NCDS taking age 23 as the baseline for analysis around $77.6 \%$ of respondents had drinking records at age 23 and 33 and $68.1 \%$ up to 43 . In the BCS around $83.4 \%$ of those with drinking records at age 26 had records at 30 and $70.5 \%$ up to age 34 . The BCS suffered from greater attrition than the NCDS particularly at age 26 where participants had to opt in by sending through a postal questionnaire. Complete cases were not lower than $90 \%$ of those with drinking records at consecutive waves. 


\section{Rates of earlier health conditions with drinking status in the NCDS and BCS}

In the NCDS in table 1 around $82.2 \%$ of respondents with drinking records at age 23 had medical records at age 16 and this did not appear to differ significantly by drinking group (Table 1). Where there was a statistical difference between drinking status and rates of abnormalities, non-drinkers had the highest rates of speech $(5.7 \%)$, mental disability $(9.8 \%)$, emotional and behavioural problems (6.5\%), physical disability (9.5\%), epilepsy (2.4\%), heart (3.2\%) and other central nervous system conditions (2.1\%).

\section{Changes in LLSI and persistent non-drinking from age 23 in the NCDS}

In table 2 using the NCDS, those who had a limiting longstanding illness in successive waves from age 23 had 4.50 times the odds $(95 \% \mathrm{Cl} 1.99$ to $10.18, p<0.001)$ of being a lifetime abstainer at age 33 and 7.02 times the odds (2.39 to 20.66, $p<0.001)$ at age 42 adjusting for all factors. In addition having a LLSI in at least one wave before the age of the model since age 23 years was positively associated with being a lifetime abstainer at age 33 (1.57 to $5.08, \mathrm{p}<0.001)$ and $42(1.73$ to $5.77, \mathrm{p}<0.001)$. Currently having a LLSI whether the respondent had one previously or not, was associated with being a lifetime abstainer at age 42 only $(1.33$ to $3.45, p<0.01$ ). People who were separated and divorced at age 33 were less likely than those who were single to have been a lifetime abstainer $(0.23$ to 0.99 , $p<0.05)$. Women were statistically significantly more likely to be lifetime abstainers in every model $(p<0.001)$.

\section{Changes in LSI and persistent non-drinking from age 26 in the BCS}

In table 3 using the BCS people who had a longstanding illness in all time sweeps since age 26 had 2.80 times $(1.88$ to $4.18, p<0.001)$ the odds of someone who never had a $L S I$ of being a lifetime abstainer at age 30 , and 3.33 times the odds $(2.01$ to $5.53, p<0.001)$ at age 34, whilst adjusting for all factors.. 


\section{Changes in LSI and those who reported 'never had an alcoholic drink' from age 30 in the BCS}

Similarly for those who reported "never having an alcoholic drink" in table 4 , those who had a LSI in all waves had 2.11 times $(1.24$ to $3.57, p<0.01)$ the odds of someone who never had a LSI of being a self-reported lifetime abstainer at age 30 and 2.80 times the odds at age 34 (1.62 to $4.84, p<0.01$ ) Having previously had a LSI but not currently was also significantly associated with being a never ever drinker at age 30 (OR 2.07, 1.08 to $3.98, p<0.05$ ) and 34 (OR 2.02, 1.20 to $3.42, p<0.001$ ). Having children was also associated with being a lifetime abstainer at age 30 (OR 1.89, 1.21 to 2.97, $\mathrm{p}<0.01)$. Gender had no effect in each of the BCS models.

\section{Discussion}

Non-drinkers have been found to have higher rates of self-reported illness in young adulthood $(10,11)$ however this is the first paper to show that objectively assessed medical conditions at 16 years are more common among non-drinkers in their twenties and that persistent self-rated poor health was associated with staying a non-drinker over adulthood. In addition people who reported having a self-reported illness over consecutive waves were more likely to have remained non-drinkers over the same time waves. This was the case for both LLSI in the NCDS from 23 to 42 years and LSI in the BCS from 26 to 34 years. Consistent evidence between cohorts provides strong support that ill health is associated with continued non-drinking at different stages of the life-course particularly if the condition is persistent. Furthermore an association with both LSI and LLSI shows that it is not only people with severe illness or with disability that do not drink alcohol however further analysis should be carried out to assess whether more severe health conditions have a greater effect on general alcohol consumption.

We also found associations between persistent LSI and those who consistently stated they were non-drinkers in each wave and participants who reported being lifetime abstainers in 
the BCS at 30 and 34 years providing two different methods of deriving lifetime abstainers. This implies that illness is associated with both those who consistently say they are nondrinkers over time and those who claim to be lifetime abstainers, which has implications for either as a control group against drinkers, where the latter has already been found to be subject to validity issues as those who claim to have never ever drank alcohol were found to report drinking in previous surveys $(12,13)$.

Additionally having previously had a LLSI but not having one currently was also significantly associated with lifetime abstention in the NCDS at 33 and 43 years, and with self-reported lifetime abstainers in the BCS, which supports the argument that some people never ever take up drinking because of ill health at an early age. This may reflect people who never start drinking due to illness and continue to abstain even once health improves, particular when an early age of onset of drinking has been shown to have to influence of drinking in adulthood (16). Alternatively a condition may have improved with medication however continued use of medication prohibits the use of alcohol indicating there may be a time lag in which health effects alcohol consumption. Unfortunately we are unable to verify this with the dataset.

Evidence shows that non-drinkers have poorer psychosocial health than drinkers $(17,18)$ including in early adulthood $(10,19-21)$. It is possible that prior health conditions may be confounding the relationship between poorer mental health and non-drinking found in other studies as to our knowledge this is the first study to combine LSI and psychosocial health measured by the malaise inventory. In this study however, whilst we controlled for a measure of psychosocial health, we cannot rule out that mental health may actually be a limiting longstanding illness although LSI has been found to have stronger associations with physical functioning than mental and social wellbeing $(22,23)$ while another study using the NCDS found that the stability of limiting longstanding illness in early adulthood across a decade was associated with prior diagnoses of disability in childhood (23). 
Reasons for why poor health has found to be associated with non-drinking in this study and others $(8,9,11)$, may be due to the direct impact on drinking because of the health condition or interference with medication. Many medications interact harmfully with the consumption of alcohol prohibiting the use of alcohol, including medications for epilepsy and muscular pain (24) ailments which were found to be more common among non-drinkers in early adulthood (Table 1). This may be why associations of poor health and non-drinking to be present across the life course as we find in this study and may also contribute to explaining the sick quitter phenomenon. Another important consideration is the social gradient in nondrinking where people with lower incomes and less education are more likely to be nondrinkers $(11,25-27)$. Low social economic status, poor health and mental health all interrelate and contribute to disadvantage and deprivation resulting in higher morbidity and may suggest interdependent and indirect effects of each on non-drinking through social exclusion.

Rates of lifetime abstainers did not differ greatly between cohorts which may represent a stable and steady group among the drinking population. Women were more likely to be lifetime abstainers in every regression model in the NCDS, however among the younger cohort in the BCS gender had no effect. This may be a consequence of more acceptable drinking norms for women where drinking rates have risen among women over the past decades. Unfortunately due to the low sample size of lifetime abstainers we were unable to stratify by gender. For future studies the effects of health on drinking should be assessed in populations where drinking is less of a norm. In such populations we imagine the effect of health to be lower among non-drinkers due to the higher number of 'average' people among non-drinkers where drinking is less normative.

The strengths of the study include the use of two large nationally representative cohort studies and finding consistent results particularly with the association between persistent LLSI and LLSI and remaining a non-drinker. Limitations of this paper include missing data. Since we used complete case analysis that depended on data from consecutive waves there 
was a large proportion of missing data, which was largely down to attrition (figure 1) We find no statistical variation between missing data at age 23 and medical records at age 16 in the NCDS (table 1) and consistent findings between two cohorts. We also cannot rule out an element of missing not at random when concerning limiting longstanding illness as some may have been too ill to participate in the next wave. However if our hypothesis that nondrinking is a consequence of poor health is true than this would underestimate the associations due to a reduced sample from people who were very ill, however we are unable to verify this.

\section{Conclusion}

Existing health problems from earlier life or chronic health problems during adulthood may be a reason why some people will not take up alcohol across their life course. Even lifetime abstainers may suffer from pre-existing poor health and therefore the better health outcomes of moderate drinkers compared with non-drinkers may be subject to bias. Care should be taken in verifying previous and existing health status of drinking groups in studies which aim to examine the short or long-term effects of alcohol on adult health.

\section{What is already known on this topic}

A J-shape relationship is observed between alcohol consumption and mortality outcomes with moderate drinkers having better health and mortality outcomes than non-drinkers.

Lifetime abstainers have been used as a reference group to eliminate bias resulting in including ex-drinkers who may have quit drinking due to worse health.

It is not known whether poor health from an early age or on-going poor health may be a reason why some people never ever take up drinking.

\section{What this study adds}

Objectively assessed medical conditions at age 16 were higher among non-drinkers in early adulthood.

Having a limiting longstanding illness or longstanding illness consistently across adulthood is a predictor of being a lifetime abstainer independent of social, demographic and psychosocial health factors. 
Illness from an early age and on-going illness may be a reason why some never ever take up drinking alcohol. Studies which compare the health of moderate drinkers with lifetime abstainers may be subject to a pre-existing poor health bias.

\section{Footnotes}

- Licence for Publication

The Corresponding Author has the right to grant on behalf of all authors and does grant on behalf of all authors, an exclusive licence (or non exclusive for government employees) on a worldwide basis to the BMJ Publishing Group Ltd to permit this article (if accepted) to be published in JECH and any other BMJPGL products and sublicences such use and exploit all subsidiary rights, as set out in our licence (http://group.bmj.com/products/journals/instructions-for-authors/licence-forms).

- Competing interests: None declared

- Contributor: LN and NS had the original idea for the study and developed the study design. LN conducted all analyses and wrote the first draft. NC and NS advised on statistical aspects of the study design, analysis stages and revision of drafts. All authors were involved in discussion and interpretation of results. LN is guarantor for the study.

- Funding: Alcohol Research UK (ARUK) and UCL Impact provided a scholarship to fund LN's PhD. The funding body played no part in the research protocol, data analyses, data interpretation, or writing of the report. TThe authors' work is independent of the funders. The views expressed in this article are those of the authors and do not necessarily reflect those of the funders.

- Ethical approval: Ethical approval was obtained from relevant approval bodies in the UK at the time of data collection. Access to the dataset for the purposes of secondary analysis was subject to the terms of an end user license agreement, and further ethical approval was not needed.

- Data sharing: The technical appendix and statistical code are available from LN at I.ngfat.10@ucl.ac.uk. The data are available from the Economic and Social Data Service (http://www.esds.ac.uk) subject to an end-user license agreement.

\section{References}

1. Marmot M, Brunner E. Alcohol and cardiovascular disease: the status of the $U$ shaped curve. British Medical Journal. 1991(303):565-8.

2. Corrao G, Rubbiati L, Bagnardi V, et al. Alcohol and coronary heart disease: a meta-analysis. Addiction. 2000;95(10):1505-23.

3. Ronksley PE, Brien SE, Turner BJ, et al. Association of alcohol consumption with selected cardiovascular disease outcomes: a systematic review and meta-analysis. British Medical Journal. 2011;342(d671).

4. Di Castelnuovo A, Costanzo S, Bagnardi V, et al. Alcohol Dosing and Total Mortality in Men and Women: An Updated Meta-analysis of 34 Prospective Studies. Arch Intern Med. 2006;166(22):2437-45.

5. Anstey $\mathrm{K}$, Mack $\mathrm{H}$, Cherbuin N. Alcohol consumption as a risk factor for dementia and cognitive decline: meta-analysis of prospective studies. American Journal of Geriatric Psychiatry. 2009;17(7):542-55. 
6. Shaper AG, Wannamethee G, Walker M. Alcohol and Mortality in British men: Explaining the U-shaped curve. The Lancet. 1988;332(8623):1267-73.

7. Wannamethee SG, Sharper AG. Lifelong teetotallers, ex-drinkers and drinkers: mortllity and the incidence of major coronary heart disease events in middle-aged British men. International Journal of Epidemiology. 1997;26:523-31.

8. Liang W, Chikritzhs T. Reduction in alcohol consumption and health status. Addiction 2011(106):75-81.

9. Newsom JT, Huguet N, McCarthy MJ, et al. Health Behavior Change Following Chronic Illness in Middle and Later Life. The Journals of Gerontology Series B: Psychological Sciences and Social Sciences. 2012;67B(3):279-88.

10. Power C, Rodgers B, Hope S. U-shaped relation for alcohol consumption and health in early adulthood and implications for mortality. The Lancet. 1998;352(9131):877-.

11. Ng Fat L, Shelton N. Associations between self-reported illness and non-drinking in young adults. Addiction. 2012;107(9):1612-20.

12. Caldwell TM, Rodgers B, Power C, et al. Drinking histories of self-identified lifetime abstainers and occassinoal drinkers: findings from the 1958 Brtisih Birth Cohort Study. Alcohol \& Alcoholism. 2006;41(6):650-4. Epub 2006.

13. Rehm J, Irving $\mathrm{H}, \mathrm{Ye} \mathrm{Y}$, et al. Are Lifetime Abstainers the Best Control Group in Alcohol Epidemiology? On the Stability and Validity of Reported Lifetime Abstention. American Journal of Epidemiology. 2008;168(8):866-71.

14. Giordano GN, Lindstrom M. The impact of changes in different aspects of social capital and material conditions on self-rated health over time: A longitudinal cohort study. Social Science \&amp; Medicine. 2010;70(5):700-10.

15. Rodgers B, Pickles A, Power C, et al. Validity of the Malaise Inventory in general population samples. Social Psychiatry and Psychiatric Epidemiology. 1999;34(6):333-41.

16. Pitkänen T, Lyyra A-L, Pulkkinen L. Age of onset of drinking and the use of alcohol in adulthood: a follow-up study from age 8-42 for females and males. Addiction. 2005;100(5):652-61.

17. Lucas N, Windsor TD, Caldwell TM, et al. Psychological Distress in Non-Drinkers: Associations with Previous Heavy Drinking and Current Social Relationships. Alcohol and Alcoholism. 2010;45(1):95-102.

18. Saarni SI, Joutsenniemi K, Koskinen $\mathrm{S}$, et al. Alcohol consumption, abstaining, health utility, and quality of life - a general population survey in finland. Alcohol and Alcoholism. 2008;43(3):37686.

19. Caldwell TM, Rodgers B, Jorm AF, et al. Patterns of association between alcohol consumption and symptoms of depression and anxiety in young adults. Addiction. 2002;97(5):58394.

20. Leifman $\mathrm{H}$, Kühlhorn $\mathrm{E}$, Allebeck $\mathrm{P}$, et al. Abstinence in late adolescence--Antecedents to and covariates of a sober lifestyle and its consequences. Social Science \& Medicine. 1995;41(1):113-21.

21. Pape H, Hammer T. Sober adolescence-Predictor of psychosocial maladjustment in young adulthood? Scandinavian Journal of Psychology. 1996;37(4):362-77.

22. Cohen G, Forbes J, Garraway M. Interpreting self reported limiting long term illness. BMJ. 1995;16(311):722-4.

23. Power C, Li L, Manor O. A prospective study of limiting longstanding illness in early adulthood. International Journal of Epidemiology. 2000;29(1):131-9.

24. National Institute on Alcohol Abuse and Alcoholism. Harmful interactions: mixing alcohol with medicines. 2007.

25. Barbara J M H Jefferis, Orly Manor, Power C. Social gradients in binge drinking and abstaining: trends in a cohort of British adults. Journal of Epidemiology Community Health. 2007;61:150-3. 
26. Pattenden $S$, Nanchahal $K$, Primastesta $P$, et al. Self-reported never-drinkers in England 1994-2003: Charactersitcs and trends in adults aged 18 to 54 years. Alcohol \& Alcoholism. 2008;43(1):91-6.

27. Bloomfield K, Grittner U, Kramer S, et al. Social inequalities in alcohol consumption and alcohol-related problems in the study countries of the EU Concerted action 'Gender, Culture and Alcohol problems: A multi-national study'. Alcohol \& Alcoholism. 2006;41(1):i26-i36. 